\title{
Spatiotemporal Sensitivity following Lesions of Area 18 in the Cat
}

\author{
Tatiana Pasternak and John H. R. Maunsell \\ Departments of Neurobiology and Anatomy, and Physiology, and Center for Visual Science, University of Rochester, \\ Rochester, New York 14627
}

\begin{abstract}
The contribution of cat area 18 to spatiotemporal sensitivity and to motion processing was assessed in cats with unilateral ibotenic acid lesions placed in physiologically identified portions of area 18. The lesions were centered in the representation of the lower right visual field, about $10^{\circ}$ from the vertical meridian. In one of the animals, the lesion invaded a small portion of area 19. We measured detectability of various spatiotemporal stimuli placed within the lesioned and intact portions of the visual field, while monitoring eye position with a scleral search coil. We found a loss of sensitivity to gratings of low and intermediate spatial frequency, within the ablated portion of the visual field. The sensitivity loss was $0.6-1.0 \mathrm{log}$ units at low and intermediate spatial frequencies, and decreased at higher frequencies with the resolution limits remaining intact. The loss extended over a range of temporal frequencies for both drifting gratings and gratings modulated in counterphase. We also found that within the lesioned hemifield, the cats were unable to discriminate between rightward and leftward motion even at the highest contrasts. These results demonstrate that area 18 plays an important role in detecting drifting low- and intermediate-spatial-frequency targets and is likely to represent a critical stage in the cortical processing of motion signals.
\end{abstract}

Neurophysiological studies revealed important differences between the two major visual cortical areas in the cat, areas 17 and 18 . Neurons in area 17 prefer relatively high spatial frequencies and respond well to very low temporal frequencies. On the other hand, neurons in area 18 prefer lower spatial frequencies and respond poorly to very low temporal frequencies (Movshon et al., 1978; Berardi et al., 1982; Bisti et al., 1985), and a larger proportion of them are directionally selective (Orban et al., $1981 \mathrm{~b}$ ). As a result, neurons in the two areas respond to a different range of stimulus speeds, with area 17 neurons preferring substantially lower drift rates than neurons in area 18 (Orban et al., 1981 a; Berardi et al., 1982). Another distinctive feature of area 18 neurons is greater receptive field size (Galli et al., 1988), and a decrease in the preferred spatial frequency (Bisti et al., 1985) in response to high-temporal-frequency gratings (Galli et al., 1988).

\footnotetext{
Received Oct. 1, 1991; revised June 9, 1992; accepted Junc 18, 1992.

This work was supported by grants from the National Eye Institute, EY06175, EY0591 1, and EY01319 (Core Grant to the Center for Visual Science). We thank Marc Dorfman, Kathy Lazaroff, Judy Tompkins, Robert Sikora, Larry Ota, and Peter Vamvakias for excellent technical assistance. We also thank William Merigan and Carl Olson for comments on the manuscript.

Correspondence should be addressed to Tatiana Pasternak, Center for Visual Science, University of Rochester, Rochester, NY 14627.

Copyright (C) 1992 Society for Neuroscience $0270-6474 / 92 / 124521-09 \$ 05.00 / 0$
}

Comparison of the spatiotemporal properties of area 18 neurons with properties of motion mechanisms of cats revealed in psychophysical studies shows striking parallels: motion is detected and discriminated best under stimulus conditions that are optimal for neurons in area 18 , that is, when targets move briskly and are of low spatial frequency (Pasternak and Leinen, 1986; Pasternak, 1987).

These parallels, as well as the high incidence of directionally selective neurons, suggest that area 18 represents an important stage in motion processing. We used lesions to examine the separate contribution of area 18 to visual function. Such an approach is complicated by the proximity of areas 17 and 19 and, in particular, by the confluence of representations of area centralis in areas 17 and 18 (Tusa et al., 1979). We solved these problems by limiting lesions to a portion of area 18 that represents more peripheral visual field, and by comparing visual performance in lesioned and nonlesioned visual field. Within the locus of area 18 lesions, we found a severe loss of sensitivity to low and intermediate spatial frequencies but intact spatial resolution. Moreover, such lesions abolished the ability to discriminate grating direction. These results demonstrate the critical role of area 18 in the processing of spatiotemporal frequencies that are important for motion perception and in the ability to discriminate motion direction.

\section{Materials and Methods}

Subjects. Two adult female cats were used. During the period of behavioral testing, they were maintained at $75-80 \%$ of their normal body weight. Water was continually available in the home cage, and they received a daily supplement of Purina Chow.

Stimuli. Drifting, counterphase, or stationary gratings were displayed on a 1332A HP monitor ( $\mathrm{P}-31$ phosphor) at a distance of $42 \mathrm{~cm}$. The display was visible through a $6^{\circ}$ diameter circular aperture in a large $\left(60^{\circ}\right)$ white surround. The mean luminance of the display and its surround was set to $40 \mathrm{~cd} / \mathrm{m}^{2}$. Stimulus duration was $450 \mathrm{msec}$ and followed a $200 \mathrm{msec}$ raised cosine temporal envelope.

Control of eye position. The details of procedures have been described elsewhere (Pasternak and Horn, 1991). Briefly, eye position was monitored with magnetic search coils (Fuchs and Robinson, 1966; Remmel, 1984). A scleral search coil and head restraint device were implanted after the cat had adapted to the apparatus and learned the basic behavioral task. During behavioral testing, each cat was placed in a magnetic field generated by a $50 \mathrm{~cm}$ field coil (Remmel, 1984) and its head was held firmly by means of small rods inserted through metal cylinders. Eye position was calibrated prior to each daily psychophysical session by rewarding the cat for fixating a dim laser spot projected onto the display surround. When the fixation spot appeared, the cat was required to position its ga2c within a window centered on the target (Sparks and Sides, 1974) and maintain fixation within this window (usually $\pm 1^{\circ}$ ) for a specified period of time (usually $700-1000 \mathrm{msec}$ ) in order to obtain food reward. The fixation spot was moved randomly into one of three positions along the horizontal meridian $\left(-10^{\circ}, 0^{\circ},+10^{\circ}\right)$, and the position signal from the coil was compared to target position. The coil system was adjusted after the cat reliably positioned its eye relative to 
the target. Approximately 10-20 trials were usually needed for this calibration.

Psychophysical procedure. Each trial began with the appearance of the fixation spot. When the cat fixated within $\pm 2^{\circ}$ of the spot and maintained fixation for $700 \mathrm{msec}$, a grating or a uniform field signaled by a tone was presented. After the termination of the tone $(450 \mathrm{msec})$, the cat was allowed to make a response. In the detection task, pressing the right pedal after the grating presentation, or the left pedal after the presentation of the uniform field, was rewarded with pureed beef. In the $d i$ rection discrimination task, the right pedal response was rewarded if the grating moved to the right, and a response on the left pedal was rewarded if the grating moved leftward. An incorrect response was followed by a $6 \mathrm{sec}$ loud tone and no reward. A break in fixation or a response during stimulus (or blank) presentation resulted in the termination of the trial. Responses during the $5 \mathrm{sec}$ intertrial interval delayed the beginning of the next trial. The sequence of grating/blank (or rightward/leftward motion) presentation was randomized from trial to trial. To avoid position biases, a correction procedure was used: after three consecutive errors on one pedal, the same trial was repeated until the animal made a correct response (which was not included in the data analysis). Sessions in which detection sensitivity was measured consisted of 250 trials, of which $40-50 \%$ were blank trials during which a uniform field was presented. Direction discrimination sessions consisted of 200 trials.

Threshold measurements. Thresholds were measured after the cat reached criterion performance (four consecutive sessions $>80 \%$ correct or three sessions $>90 \%$ correct) on the task requiring discrimination between a high-contrast moving grating and a uniform field (or highcontrast gratings moving in opposite directions). A staircase procedure was used to measure thresholds. In the detection task, three correct responses to the presentation of the grating produced a decrease in contrast by $0.08 \log$ units (or increase in spatial frequency by 0.14 octaves), and each incorrect response to the grating presentation made the stimulus more detectable, either by an increase in contrast or a decrease in spatial frequency. During blank trials, the correct response was rewarded and the incorrect response was followed by a loud tone, but the cat's performance had no effect on the staircase. The animal's performance during blank trials averaged about $80-90 \%$ correct (i.e., $10-20 \%$ false alarms). A comparable procedure was employed in the direction discrimination task with the exception that there were no blank trials and responses on either of the pedals produced a change in staircase value. These procedures ensured that the animal's performance was maintained at about $77 \%$ correct. Thresholds were taken as the stimulus value corresponding to $75 \%$ correct calculated by combining responses to stimuli and associated blanks. At least three or four threshold determinations were made for each stimulus condition. Threshold measurements were performed at various eccentricities. When the target was viewed centrally, the fixation spot was placed at the center of the display. For peripherally viewed targets, eccentricity was taken as the distance between the fixation spot and the center of the display. Cat 144 viewed the targets binocularly, while for cat 142 an occluder was placed in front of the right eye, and all threshold measurements and eye position calibrations were done for the left eye alone. Monocular testing was necessary due to a slight misalignment between the two eyes. This misalignment made it difficult to obtain a reliable reading of the eye position when both eyes were used.

Cortical lesions. Lesions were made following physiological mapping of the area to be ablated. The animal was initially sedated with ketamine $(15 \mathrm{mg} / \mathrm{kg})$, and the trachea and a leg vein were cannulated. The animal was then anesthetized with pentobarbital and placed in a stereotaxic apparatus. A craniotomy was made over visual cortex on the right side. The dura mater was left intact and was covered with agar in sterile solution. The cat was then paralyzed (Pavulon or gallamine), artificially respired at a rate that kept end-tidal $\mathrm{CO}_{2}$ near $32 \mathrm{mmHg}$, and maintained on an intravenous infusion for the remainder of the session (pentobarbital, $1.25 \mathrm{mg} / \mathrm{kg} / \mathrm{hr}$, and Pavulon, $5 / \mathrm{mg} / \mathrm{kg} / \mathrm{hr}$, or gallamine, $7.5 \mathrm{mg} /$ $\mathrm{kg} / \mathrm{hr}$ ). Body temperature was held near $37^{\circ} \mathrm{C}$ with a thermostatically controlled water pad. EKG, $\mathrm{CO}_{2}$, and temperature were continually monitored throughout the session.

The eyes were treated with atropine and Neo-Synephrine, and fitted with contact lenses to focus them on a tangent screen $114 \mathrm{~cm}$ from the animal. Each lens had an artificial pupil with a diameter of $3 \mathrm{~mm}$. The location of the optic disk was plotted on the screen by use of a reversing ophthalmoscope. Multiunit recordings were made using glass-insulated $\mathrm{Pt} / \mathrm{Ir}$ microelectrodes. Signals were amplified, filtered, and monitored with an oscilloscope and audio amplifier. Receptive fields were plotted on the tangent screen using a hand-held projector. After identifying the region of area 18 to be ablated, ibotenic acid $(10 \mu \mathrm{g} / \mu \mathrm{l})$ was injected using a $10 \mu \mathrm{l}$ Hamilton syringe. Four closely spaced $0.75 \mu \mathrm{l}$ injections were made in each animal.

Behavioral testing resumed about 1 week after the lesion and continued for a period of 24 months.

Histology. At the conclusion of behavioral testing, one animal (cat 142) was prepared for an acute physiological recording session with the aim of mapping the extent of the lesion. Unfortunately, the surgical procedures related to making the lesion had produced dural adhesions that resulted in cortical trauma when the old craniotomy was opened. We were unable to obtain useful physiological recordings. We did not attempt physiological mapping of the lesion in the second animal.

Each animal was killed with an overdose of barbiturate and perfused with a phosphate buffer rinse followed by $4 \%$ paraformaldehyde fixative. A second rinse was used to removed excess fixative. The brain was removed, blocked, and sectioned at a thickness of 32 or $40 \mu \mathrm{m}$. Pairs of adjacent sections at intervals of 0.25 or $0.50 \mathrm{~mm}$ were stained for cytochrome oxidase (Wong-Riley, 1979) or with cresyl violet.

\section{Results}

\section{Cortical lesions}

The ibotenic acid injections resulted in lesions with well-defined borders. In sections stained for cytochrome oxidase, little or no reactivity was present, while sections stained with cresyl violet showed no neuronal cell bodies. A typical example of the lesion in one of the cats is shown in a coronal histological section in Figure 1. This section was stained for Nissl substance, and the lesion (arrow) is visible as a lightly staining region reaching into the medial bank of the lateral sulcus. Figure 2 shows tracings of sections from the lesion in cat 144. The lesion extended to the fundus of the lateral sulcus. The border with area 19 is indicated in the section slightly posterior to the lesion (top left) and was determined from the distinct appearance of layer 4 in areas 17 and 18 (Price, 1985). Comparing the location of the lesion to the published maps (Tusa et al., 1979) and recordings made at the time of injection, we estimate that the damage involved eccentricities ranging from $5^{\circ}$ to $20^{\circ}$ azimuth and between $-2^{\circ}$ and $-8^{\circ}$ elevation. At this AP level, the representation of area 19 can reach onto the medial bank of the lateral sulcus. This representation could extend from $5^{\circ}$ to $10^{\circ}$ azimuth and down trom about $5^{\circ}$ in the lower visual field. Thus, it is likely that the lesion in this cat included a small portion of area 19 , and we cannot rule out the possibility that some behavioral testing involved portions of the visual field affected by this damage.

The histological sections from the other animal, in which physiological mapping was attempted (see Materials and Methods), were suboptimal. It was however, possible to locate the lesion in sections stained for Nissl substance. The lesion in this cat was more medial than that in cat 144 , and invaded area 17 for about $2 \mathrm{~mm}$. The damage to area 17 is unlikely to be of consequence since this portion of area 17 represents the portion of the visual field within a few degrees of the vertical meridian (Tusa et al., 1979), and behavioral testing in this animal was done beyond $5^{\circ}$ azimuth.

Figure 3 shows the placement of lesions in the two animals. The relative positions of recording sites sampled at the time the lesions were made are plotted in the left panels, and corresponding receptive field plots are illustrated in the right panels. The recording sites were clustered on the marginal gyrus at about 1 $\mathrm{mm}$ anterior in Horsley-Clarke coordinates. Sites were identified as being in area 18 on the basis of a progression of receptive fields away from the vertical meridian in moving medial to lateral (e.g., sites C-F in cat 142). Sites with receptive fields 


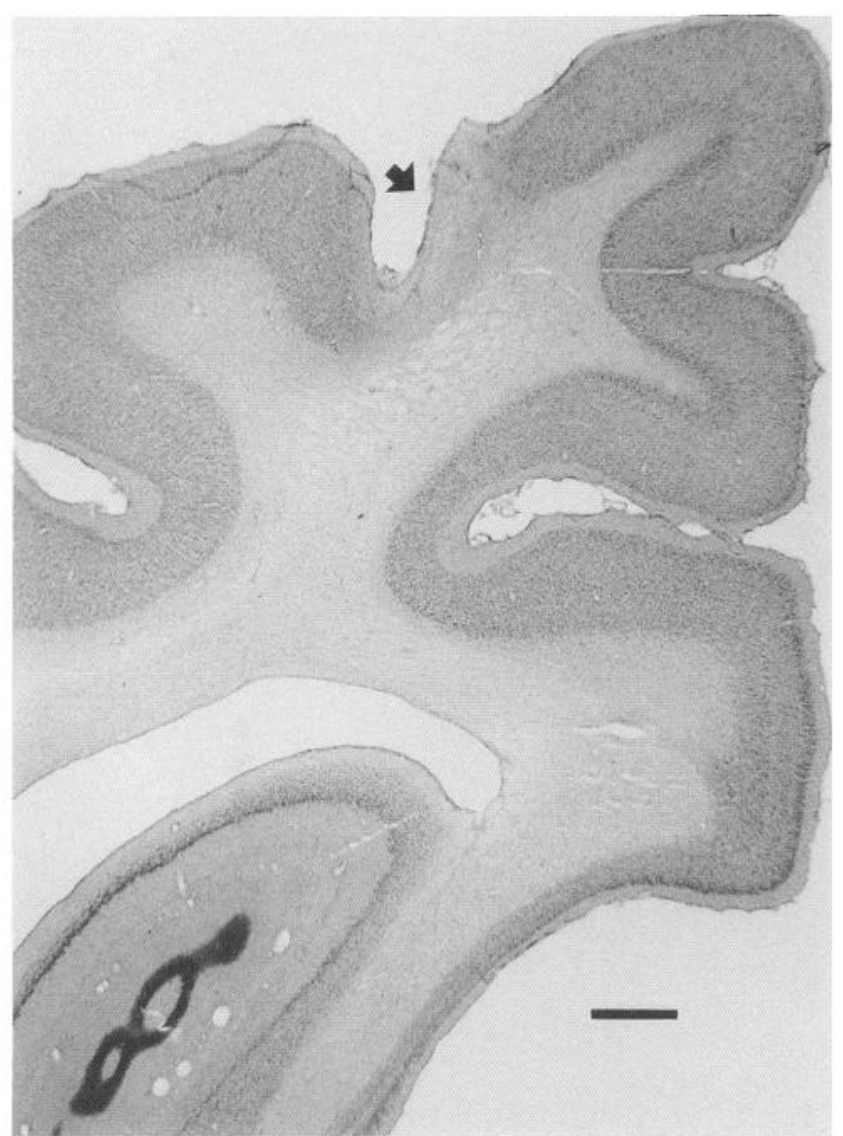

Figure 1. Histological section showing the ibotenic acid lesion in area 18 of cat 144. The section (Horsley-Clark: near A1.0) has been stained with cresyl violet. The lesion is visible as a lightly staining region extending into the medial bank of the lateral sulcus. The lesions in both animals were compact and spanned about a $2.5-\mathrm{mm}$-diameter area of cortex. Scale bar, $2 \mathrm{~mm}$.

several degrees into the ipsilateral hemifield are likely to have been near the border between areas 17 and 18. Broken lines mark the approximate location of this border. Four injections of ibotenic acid were made in area 18 of each animal, centered near representations of $5-10^{\circ}$ azimuth and $-5^{\circ}$ elevation. The injections were separated by about $1 \mathrm{~mm}$ (solid circles), and resulted in a confluent lesion. The injections ablated all cortical layers over a region of about $2-3 \mathrm{~mm}$, and destroyed a subset of cortical layers over about $4 \mathrm{~mm}$. The shaded area is an estimated extent of the visual field affected by the lesion. The estimate is based on the comparison of the histological reconstructions to the published maps and the loci of injection sites.

\section{Spatial contrast sensitivity: detection}

We first assessed the effect of the lesion by measuring contrast sensitivity for low-spatial-frequency gratings at several loci in the intact and lesioned lower hemifields. This, in conjunction with the physiological mapping performed at the time of the lesion, provided us with an estimate of the spatial extent of the lesion. The results of this psychophysical mapping are shown in Figures 4 and 5. In Figure 4, contrast sensitivity for a 0.14 cycles/degree (cat 142) or 0.3 cycles/degree (cat 144) grating drifting at $4.5 \mathrm{~Hz}$ is plotted as a function of azimuth (taken as distance from the vertical meridian to the center of the $6^{\circ}$ diameter target). The target was centered at $6^{\circ}$ and $3^{\circ}$ below the

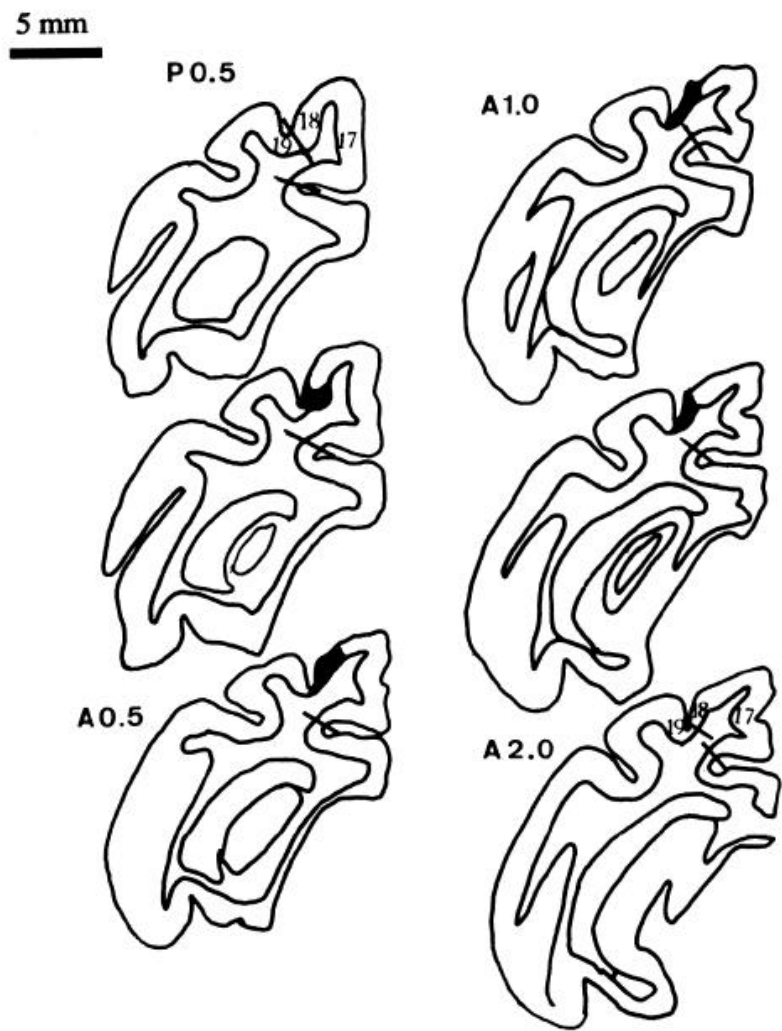

Figure 2. Drawings of coronal sections of the brain of cat 144 showing the extent of the lesion. The sections are shown at about $0.5 \mathrm{~mm}$ intervals. Drawings were made from sections stained for cytochrome oxidase in which the lesion is seen as an area of reduced reactivity that is not appreciably darker than the white matter. The lesion is shown in black and extends over about $2 \mathrm{~mm}$. The numbers indicate the approximate AP coordinates. Lines perpendicular to the sections indicating the border between areas 18 and 19 and the extent of area 17 were determined from the distinct appearance of layer 4 in sections stained for cytochrome oxidase.

horizontal meridian for cats 142 and 144 , respectively. The data for cat 142 show that sensitivity within the lesioned hemifield decreased rapidly with distance from the vertical meridian and was minimal around an azimuth of $10^{\circ}$. With greater distance, the sensitivity increased again, suggesting an outer boundary of the lesion. The sensitivity of cat 144 also decreased rapidly as the target was moved into the right hemifield. It was minimal when the target was centered on $10^{\circ}$ and increased again at $15^{\circ}$ eccentricity, suggesting that the lesion was unlikely to extend far beyond $20^{\circ}$ from the vertical meridian. Unfortunately, for this cat we were unable to obtain reliable thresholds at eccentricities beyond $15^{\circ}$ either for the intact or the lesioned hemifields. To estimate the vertical extent of the lesion, interleaved measurements were also performed at several elevations in each cat (Fig. 5). In cat 142, sensitivity loss was maximal at an elevation of $-6^{\circ}$ and in cat 144 at an elevation of $-3^{\circ}$. It is important to point out that the sensitivity measured at all locations in the lower field of the lesioned hemifields was below normal, suggesting that the representation of most of the lower quadrant was damaged. Subsequent extensive testing was carried out at a target location where the reduction of contrast sensitivity was maximal as determined by data shown in Figures 4 and 5 . The target was centered at $15^{\circ}$ right of the vertical meridian and $6^{\circ}$ below the horizontal meridian for cat 142 , and at $10^{\circ}$ from the 


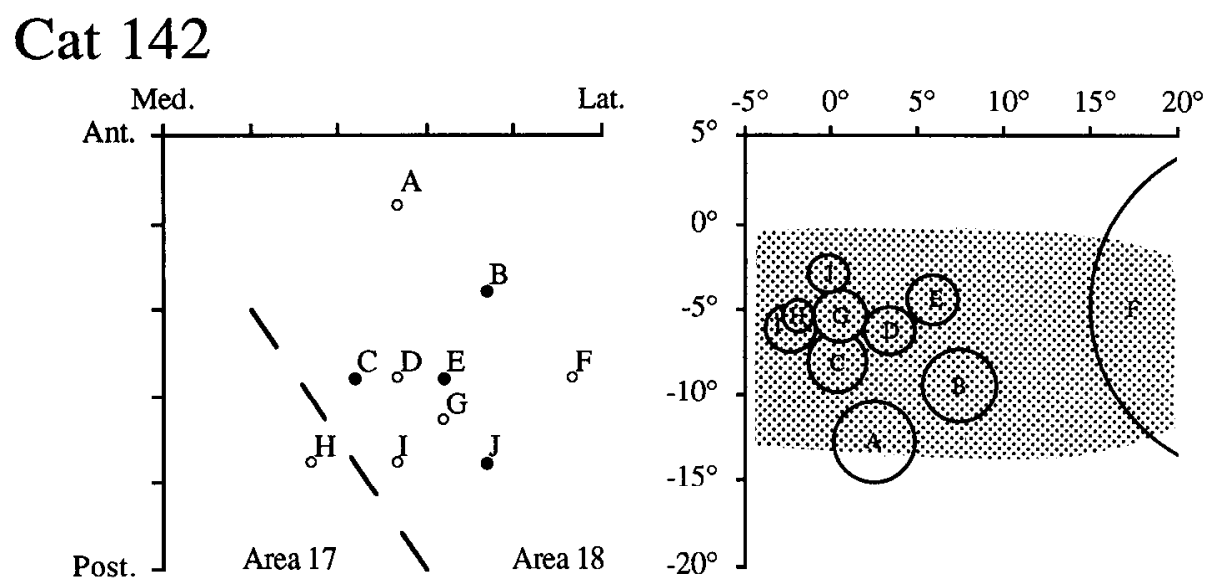

Figure 3. The placement of the lesions in area 18. The left panels show the relative positions of recording sites on the marginal gyrus that were sampled at the time the lesions were made. Tick marks are separated by $1 \mathrm{~mm}$. The right panels show corresponding receptive fields. Some sites have been omitted for clarity. Broken lines mark the approximate location of the border between areas 17 and 18 , which were determined based on receptive field progressions. The visual field representation in area 18 increases in azimuth in moving laterally across the area. Four 0.75 $\mu l$ injections of ibotenic acid were made in each animal, centered on a representation in the lower visual quadrant (solid circles). Shaded areas in right panels show the estimated extent of the visual field affected by lesions.

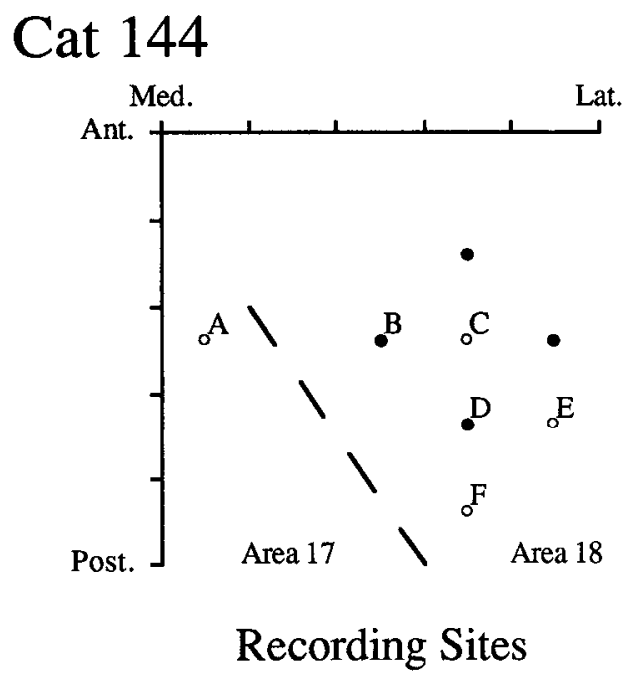

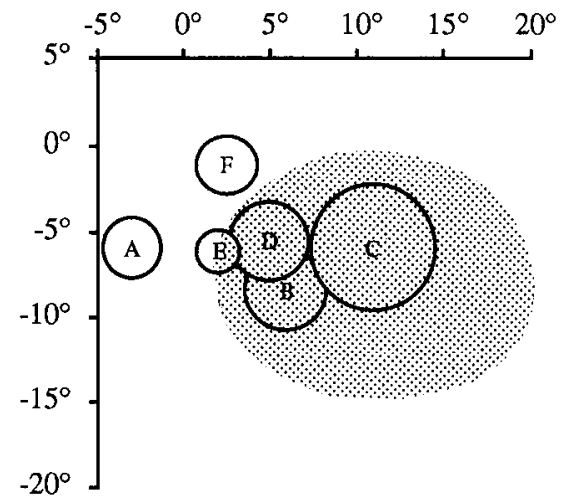

Receptive Fields vertical and $3^{\circ}$ from the horizontal meridian for cat 144 . The results of these measurements are shown in Figures 6 and 7.

Contrast sensitivity as a function of spatial frequency shown in Figure 6 was measured at symmetric loci within the intact and the lesioned hemifields. The points at the highest spatial frequency show visual acuity, which was measured separately by selting contrast to the highest obtainable value $(0.64)$ and varying spatial frequency. The data for the lesioned hemifield show greatly reduced sensitivity for the spatial frequencies ranging from 0.14 to $0.8 \mathrm{c} /$ degree. On the other hand, visual acuity measured in the lesioned hemifield was hardly affected at all.

Contrast sensitivity for low-spatial-frequency grating as a function of temporal frequency is shown in Figure 7 for the two animals. For both cats, sensitivity loss was most pronounced at the intermediate temporal frequency $(4.5 \mathrm{~Hz})$, where it ranged from 0.6 to $1.2 \mathrm{log}$ units. At the lowest temporal frequency (1.2 $\mathrm{Hz}$ ), the deficit was a bit smaller and varied from 0.4 to 0.65 $\log$ units. While in cat 144 the loss at $19 \mathrm{~Hz}$ was still quite pronounced, in cat 142 the difference between the lesioned and the intact hemifields was surprisingly small. It may be that sensitivity for the intact hemifield of cat 142 was underestimated, for much greater sensitivity was observed in cat 144 at $19 \mathrm{~Hz}$.

\section{Contrast sensitivity: direction discrimination}

In order to determine whether the residual contrast sensitivity could support the discrimination of motion direction, we mea- sured direction discrimination with low-spatial-frequency targets below the horizontal meridian. The data in Figure 8 show that performance dropped sharply when the target was placed within the lesioned hemifield, and in the location where detection sensitivity was lowest, neither of the cats was able to discriminate the direction of motion. Both cats also failed to perform the task at greater eccentricities $\left(15^{\circ}\right.$ and $20^{\circ}$ for cats 144 and 142 , respectively), even though at these locations their detection sensitivity began to improve. We have made many attempts $(5-10)$ to measure the discrimination performance within the lesioned hemifield over a period of many months. During these attempts, we explored the spatiotemporal range of the deficit, that is, used several temporal $(1.2,4.5$, and $19 \mathrm{~Hz})$ and spatial $(0.14,0.3 \mathrm{c} /$ degree $)$ frequencies. At no time the performance of the two cats exceeded $65 \%$ correct. Thus, we are convinced that in the location of maximal loss for detection, the animals were unable to discriminate motion direction.

\section{Sensitivity to counterphase flicker}

We also measured sensitivity for the detection of gratings flickered in counterphase placed within the lesioned or intact hemifield. Results obtained with drifting and flickering gratings of the same spatial and temporal frequency are compared in Figure 9. In both hemifields, the sensitivity for drifting gratings exceeded that for counterphase gratings. In the intact hemifield, the difference in sensitivity was a bit larger than that previously 

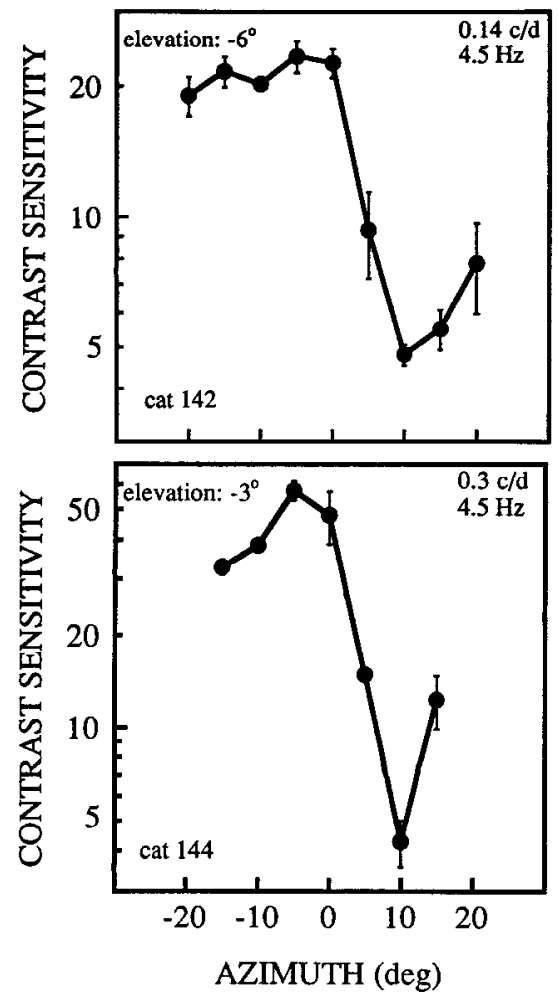

Figure 4. Contrast sensitivity for the two cats measured in several locations in the right (intact) and the left (lesioned) hemifields. For cat 142 , the target $\left(6^{\circ}\right.$ diameter) was centered at $-6^{\circ}$ and for cat 144 at $-3^{\circ}$ below the horizontal meridian. The numbers along the azimuth refer to the distance from the target center to the vertical meridian. Note that in cat 142 , the reduction in sensitivity was maximal near an azimuth of $10-15^{\circ}$, while in cat 144 , it was maximal at an azimuth of $10^{\circ}$. Error bars are SEM.

reported for humans (e.g., Levinson and Sekuler, 1975) and normal cats (Pasternak, 1986). The loss of sensitivity for counterphase gratings presented within the lesioned hemifield was similar to that for drifting gratings. Thus, the normally observed sensitivity difference for motion and flicker appears to be preserved in the absence of area 18.

The behavioral testing continued over a period of over 2 years, and we observed no improvements in performance on the tasks described above.

\section{Discussion}

We found that lesions largely limited to area 18 severely reduced detection sensitivity at low and intermediate spatial frequencies, leaving spatial resolution intact. This loss of sensitivity for detection was present at all temporal frequencies and was accompanied by a complete loss of sensitivity for discriminating motion direction.

\section{Lesion placement}

We used physiological recordings to guide the placement of our lesions and were able to make well-defined 2-3 mm lesions with relatively limited involvement of adjacent areas 17 and 19. However, in cat 142 the injections were a bit too medial and resulted in damage within the $1-2^{\circ}$ representation of the vertical meridian of area 17 . Since most of the behavioral testing was done with targets centered at $15^{\circ}$ azimuth, we are certain that damage to area 17 in this animal is very unlikely to contribute
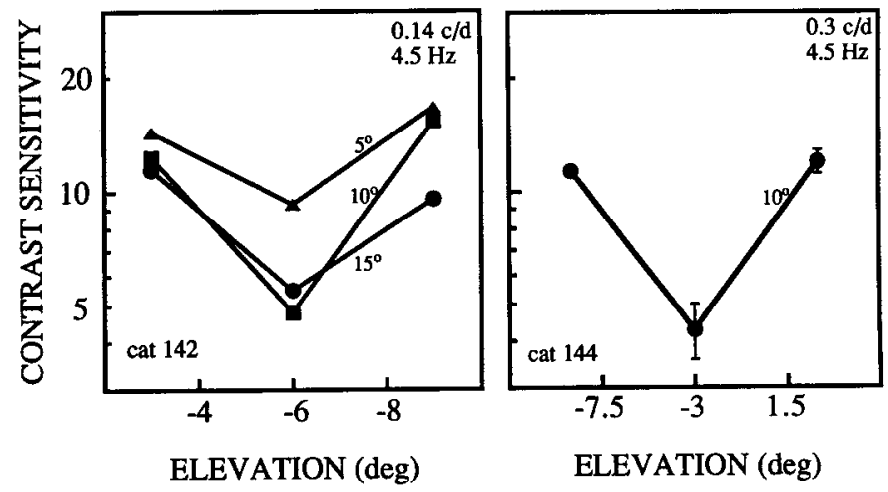

Figure 5. Contrast sensitivity for the two cats measured in the lesioned hemifield helow (cat 142), and above and below (cat 144) the horizontal meridian. The parameter near each curve indicates distance from the vertical meridian (azimuth). Measurements made along several elevations (distance from the horizontal meridian) show that maximal sensitivity loss was at $-6^{\circ}$ for cat 142 , and at $-3^{\circ}$ for cat 144 . Error bars are SEM.

to the observed deficits. In cat 144 , on the other hand, the lesion was placed a bit too lateral and, as a consequence, invaded a portion of area 19. Since the representation of the damaged portion of area 19 partially overlaps with the damaged representation of area 18 , we have to consider the possibility that the lesion effects may be partially due to the combined lesion of areas 18 and 19. Damage to area 19 in this cat, however, is an unlikely contributor to the observed deficits, since the lesion
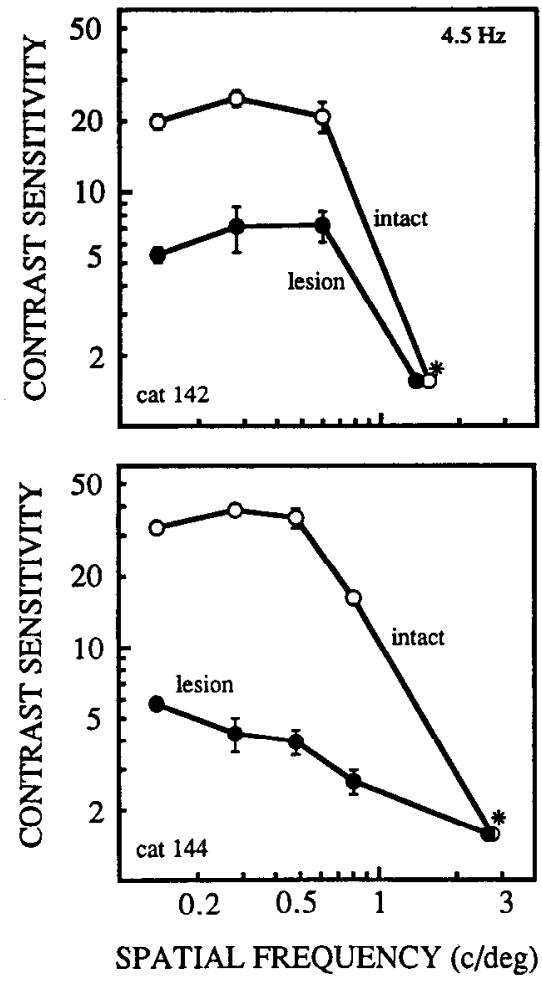

Figure 6. Spatial contrast sensitivity and acuity for a grating drifting at $4.5 \mathrm{~Hz}$ measured in the intact and the lesioned hemifields. The target was centered at $+15^{\circ}$ azimuth and $-6^{\circ}$ below the horizontal meridian for cat 142 , and at $\pm 10^{\circ}$ azimuth and $-3^{\circ}$ below the horizontal meridian for cat 144 . Acuity measurements (indicated by an asterisk) were performed by varying spatial frequency at a contrast of 0.64 . Error bars are \pm SEM. 

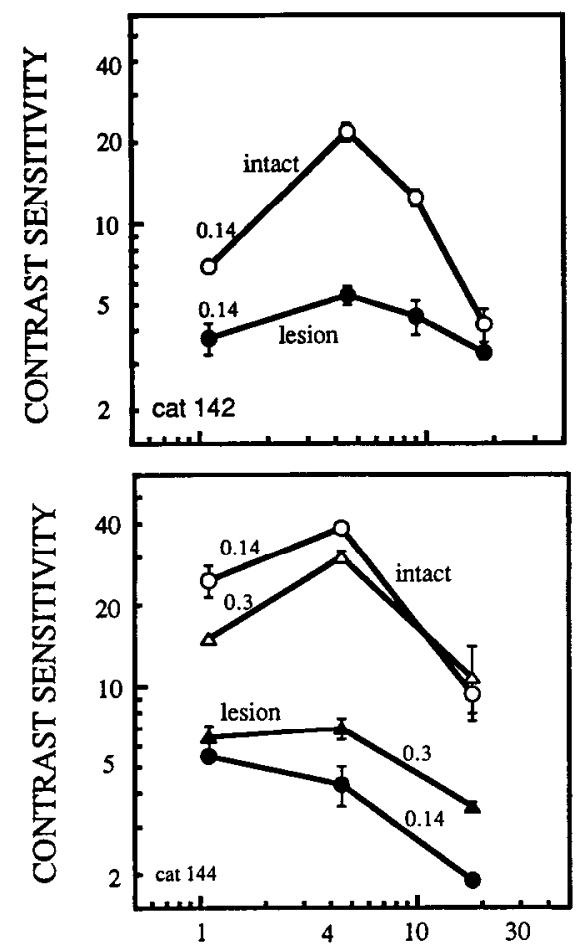

TEMPORAL FREQUENCY $(\mathrm{Hz})$

Figure 7. Contrast sensitivity for a low-spatial-frequency drifting grating as a function of temporal frequency measured in the lesioned and intact hemifields. Numbers next to each curve for cat 144 refer to spatial frequency. See Figure 6 for precise target location. Error bars are SEM.

in cat 142, which almost certainly did not include area 19, resulted in nearly identical behavioral effects. Thus, it is likely that the behavioral deficits described here are largely due to the damage limited to area 18.

\section{Spatiotemporal sensitivity}

Spatial frequency. Since neurons in area 18 respond preferentially to targets of low spatial frequency and have relatively poor spatial resolution (Movshon et al., 1978; Berardi et al., 1982), the finding that a lesion of area 18 reduces sensitivity for detection of such targets while preserving spatial resolution was not surprising. The deficit, however, extended to frequencies above $0.5 \mathrm{c} /$ degree, which is higher than would be expected from loss of area 18 alone (Movshon et al., 1978). It is possible that the loss at higher frequencies reflects a direct contribution of area 18 neurons to high-frequency sensitivity in the intact animal. Although the majority of neurons in area 18 at eccentricities beyond $10^{\circ}$ (the location of the lesions) are tuned to frequencies below $0.2 \mathrm{c} /$ degree and their bandwidth is a bit too narrow ( $<2$ octaves) to account for the detection of $0.6-0.8$ $\mathrm{c}$ /degree gratings (Movshon et al., 1978), it is possible that a small percentage of cells still respond to these frequencies and thus contribute to detection. An additional effect may be mediated by the direct projection from area 18 to 17 (Mcyer and Albus, 1981; Bullier et al., 1984; Symonds and Rosenquist, 1984; Einstein and Fitzpatrick, 1991). It is possible that the removal of area 18 affected the response properties of neurons in area 17 in these cats, and that as a result, detection sensitivity by area 17 neurons was reduced. Indeed, it has been shown that many neurons in superficial layers of area 17 can be driven

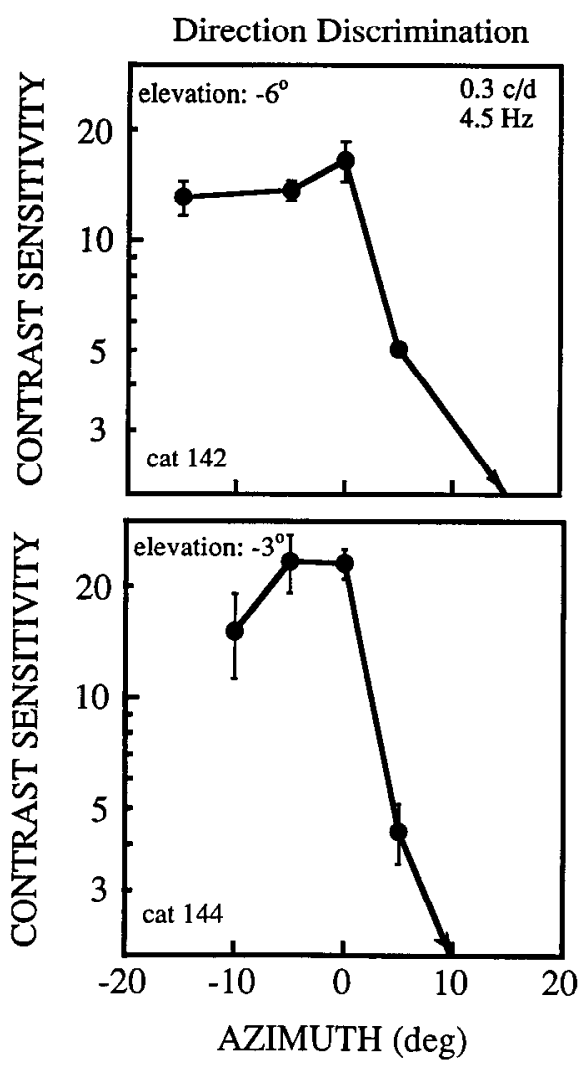

Figure 8. Contrast sensitivity for direction discrimination measured in the lesioned and intact hemifields of the two cats. The measurements were performed below the horizontal meridian at the same clcvation as those shown in Figure 3. The arrows indicate that the animals were unable to discriminate motion direction even at the highest grating contrast (0.64). Error bars are SEM.

monosynaptically by neurons in area 18 (Bullier et al., 1988) and their responses can be reduced or abolished by lesions of area 18 in cats (Nault et al., 1990; Mignard and Malpeli, 1991) and monkeys (Sandell and Schiller, 1982). Thus, while the loss of sensitivity at low spatial frequencies supports a role for area 18 in signaling these spatial frequencies, the loss at higher spatial frequencies suggests that area 18 neurons may be critical for the integrity of at least some area 17 neuronal properties.

Temporal frequency. Our results showed that the sensitivity loss for low-spatial-frequency gratings that followed area 18 lesions was present over a wide range of temporal frequencies, although the loss was most pronounced at the peak frequency for the intact hemifield, $4.5 \mathrm{~Hz}$. For the intact hemifield, a fourfold decrease in temporal frequency resulted in a substantial reduction in sensitivity, a result consistent with previous reports (Pasternak and Leinen, 1986). On the other hand, the performance of both cats in the lesioned hemifield was hardly affected by a decrease in temporal frequency. In fact, the peak sensitivity of cat 144 appears to have shifted toward lower temporal frequencies. This shift in sensitivity may reflect the removal of area 18 neurons, which respond well to higher temporal frequencies, and the survival of neurons in area 17, which, in general, respond well to low temporal frequencies (Movshon et al., 1978).

Residual spatiotemporal sensitivity. Although the lesion produced a pronounced sensitivity loss, the animals were still able to detect high-contrast targets placed within the lesioned portion 


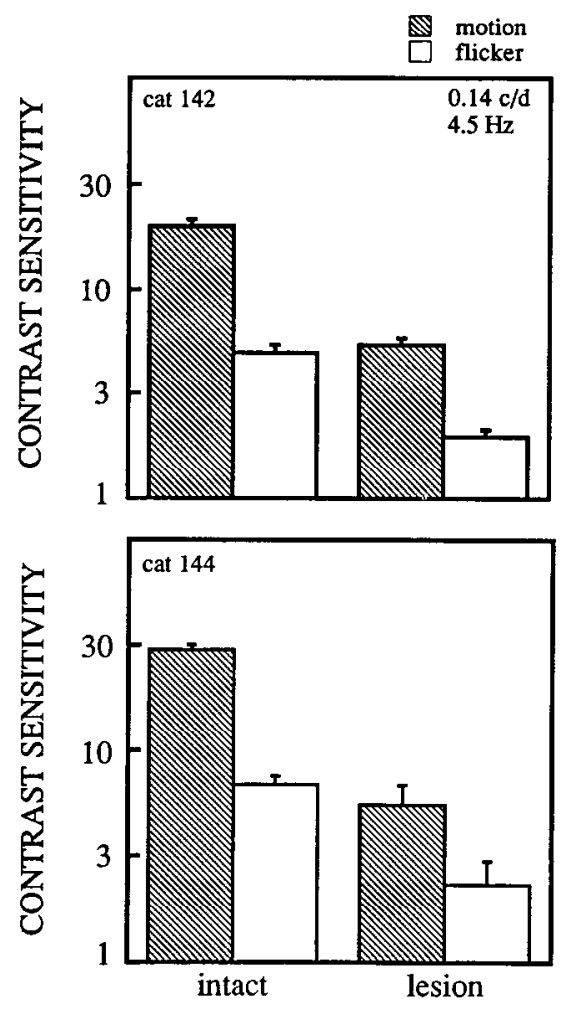

Figure 9. Contrast sensitivity for detecting drifting gratings (motion) and gratings modulated in counterphase (flicker). The measurements were performed with a los-spatial-frequency grating in the lesioned and intact hemifields. Target location is the same as that used in Figures 6 and 7. Error bars arc SEM.

of the visual field. This residual sensitivity might have been due to the extension of the target outside the lesion, with detection mediated by intact portions of area 18. This, however, is not very likely since the psychophysical and histological estimates of the extent of the lesion show that the $6^{\circ}$ target was placed well within the lesion area, which was larger than $10^{\circ}$ in diameter.

On the other hand, it is possible that the residual sensitivity reflects the response of area 17 neurons, some of which have broad enough bandwidth ( $>2$ octaves) to respond, albeit weakly, to relatively low-spatial-frequency targets (Movshon et al., 1978; Berardi et al., 1982). Furthermore, neurons in other cortical areas, such as the lateral suprasylvian (LS) area or area 19, respond well to low-spatial-frequency targets (DiStefano et al., 1985; Morrone et al., 1986; Tanaka et al., 1987; Gizzi et al., 1990). These spatiotemporal properties of LS neurons appear to survive lesions of both areas 17 and 18 (Guido et al., 1990). Similarly, responses of neurons in area 19 also appear largely unaffected by the inactivation of areas 17 and 18 (Kimura et al., 1980). Thus, it is conceivable that, in addition to area 17 , the activity of neurons in LS area or area 19 contributed to the residual sensitivity within the lesioned portion of the visual field.

Spatial resolution. The difference in sensitivity between the lesioned and the intact hemifield decreased at high spatial frequencies, with the result that visual acuity was unaffected by the lesion. We measured the acuity by requiring the cats to discriminate between a blank field and a field containing highcontrast gratings of various spatial frequencies. The visual acuity of both animals was about $1.5 \mathrm{c} /$ degree for a target centered at $16^{\circ}$ (cat 142) and $2.5 \mathrm{c} /$ degree (cat 144) for a target centered at $11^{\circ}$ eccentricity. These resolution estimates are consistent with predictions based on properties of X-cells for these eccentricities (e.g., Cleland et al., 1979; also see Pasternak and Horn, 1991). Preservation of visual acuity after area 18 lesion is consistent with the fact that cells in area 18 respond poorly to high spatial frequencies. Thus, it appears that area 18 neurons do not play an important role in spatial resolution of high-contrast targets. Evidently, signals carrying high-spatial-frequency information from area 17 do not require the integrity of area 18 to reach subsequent stages of processing.

\section{Directional mechanisms}

One of the most striking effects of area 18 lesion was an inability to discriminate the direction of motion for targets placed within the lesioned portion of the visual field. It is important to stress that we have attempted to measure direction discrimination in the lesion site over a period of several weeks with gratings drifting at speeds ranging between 1 and $19 \mathrm{~Hz}$. Each time the performance never was better than $65 \%$ correct. During the same period of time, we tested the performance on this task at other locations of the visual field and were able to get good data. Directionally selective neurons have been shown to play a critical role in the ability to discriminate opposite directions of motion (Pasternak et al., 1985; Pasternak and Leinen, 1986). Such neurons are commonly found in striate cortex as well as in extrastriate areas, including areas 18 and lateral suprasylvian cortex (Spear and Baumann, 1975; Orban et al., 1981b). Psychophysical studies have shown that discrimination of direction is possible only when the targets drift relatively fast and are of low spatial frequency (Pasternak and Leinen, 1986; Pasternak, 1990). Thus, the spatiotemporal conditions required for direction discrimination are similar to those required to stimulate optimally neurons in area 18 . This parallel, in conjunction with the high incidence of directional selectivity, suggests that area 18 is well suited for processing of motion signals. Complete loss of the ability to discriminate motion direction within the lesioned portion of the visual field strongly supports this idea. This result questions the role of directionally selective units commonly found in areas outside of area 18, such as striate cortex and several extrastriate areas. One possibility is that directional selectivity in area 17 may be secondary to that in area 18 and may be reduced as a result of inactivation of area 18 (Nault et al., 1990). It is also possible that, even if some information about target direction is present in area 17, it is not utilized, since the spatiotemporal range of the neurons providing this information is inappropriate. Another possible source of directional signals is lateral suprasylvian cortex, since directional selectivity of neurons in this area does not appear to depend on the inputs from areas 18 and 19 (Spear and Baumann, 1979). Our results suggest, however, that in the absence of area 18 , these directional signals are not used by the animal.

In normal observers, sensitivity for detecting drifting gratings exceeds that for gratings modulated in counterphase by about a factor of two (Levinson and Sekuler, 1975; Watson et al., 1980; Pasternak, 1986). This difference is thought to reflect the operation of directionally selective mechanisms (Levinson and Sekuler, 1975; Watson et al., 1980; Pasternak, 1987). We compared sensitivity to the two types of temporal modulation in order to determine whether the greater sensitivity to motion depends on area 18 neurons. The fact that the difference between 
the two types of stimuli persists within the lesioned portion of the visual field suggests that, even though the cats are unable to use directional signals to discriminate motion direction, their visual system still contains directionally selective mechanisms.

Comparison with other lesion studies. Few lesion studies have examined the role of visual cortical areas of the cat in spatial contrast sensitivity or acuity. Those that did involved lesions centered in area 17 but encroaching on area 18 (e.g., Berkley and Sprague, 1979; Lehmkuhle et al., 1982). Berkley and Sprague (1979) measured visual acuity following an extensive lesion of area 17 that also included portions of area 18, and reported a loss of visual acuity. This result might be attributable to the lesion of area 17. Lehmkuhle et al. (1982) examined spatiotemporal sensitivity following visual cortical lesions that included $30^{\circ}$ of the central representation of area 17 and the vertical meridian of area 18. The acuity loss in these animals was similar to that found by Berkley and Sprague (1979). In addition, the authors reported a relatively modest loss in sensitivity, which increased with spatial frequency. Although this pattern of loss is consistent with a lesion of area 17, damage to area 18 could also have contributed to it, particularly at lower spatial frequencies. Since fixation was not controlled, it is possible that the apparent retention of considerable sensitivity following the combined lesion was due to the cat's using portions of the visual field not affected by the lesion.

Recent studies involving ibotenic acid lesions of extrastriate cortical areas MT and MST in monkeys reported relatively large and selective behavioral deficits followed by rapid improvement in performance (e.g., Newsome and Pare, 1988; Yamasaki and Wurtz, 1991; also see Pasternak et al., 1991). In the present study, we saw no evidence of recovery during the 2 year period of postoperative testing. Similarly, no behavioral recovery was observed following ibotenic acid lesion of area V2 in monkeys (Nealey et al., 1992). It is possible that the recovery of function after lesions of relatively early stages of cortical analysis, such as area 18 in a cat or area V2 in a monkey, is much more limited than after lesions of areas that represent a later stage of cortical processing.

\section{Conclusions}

Lesions restricted to area 18 affect the detection of a relatively broad range of spatial frequencies. In fact, the range of the affected frequencies is broader than one would expect from response properties of area 18 neurons. This suggests that even though areas 17 and 18 receive distinct subcortical inputs and have different spatiotemporal properties, they are unlikely to function exclusively in parallel, with area 17 contributing to the detection only at high, and area 18 only at low spatial frequencies. Rather, in addition to playing a unique role in signaling low-spatial-frequency targets and their direction of motion, area 18 is also likely to contribute to visual sensitivity indirectly, by modulating the responses of area 17 neurons to which it projects.

\section{References}

Berardi N, Bisti S, Cattaneo A, Fiorentini A, Maffei L (1982) Correlation between the preferred orientation and spatial frequency of neurones in visual areas 17 and 18 of the cat. J Physiol (Lond) 323: 603-618.

Berkley MA, Sprague JM (1979) Striate cortex and visual acuity functions in the cat. $J$ Comp Neurol 187:679-702.

Bisti S, Carmignoto G, Galli L, Maffei L (1985) Spatial-frequency characteristics of neurones of area 18 in the cat: dependence on the velocity of the visual stimulus. J Physiol (Lond) 359:259-268.

Bullier J, Kennedy H, Salinger W (1984) Branching and laminar origin of projections between visual cortical areas in the cat. J Comp Neurol 228:329-341.

Bullier J, McCourt MC, Henry GH (1988) Physiological studies on the feedback connection to the striate cortex from cortical areas 18 and 19 of the cat. Exp Brain Res 70:90-98.

Cleland BG, Harding TH, Tulunay-Keesey U (1979) Visual resolution and receptive field size: examination of two kinds of cat retinal ganglion cell. Science 205:1015-1017.

DiStefano M, Morrone MC, Burr DC (1985) Visual acuity of neurones in the cat lateral suprasylvian cortex. Brain Res 331:382-385.

Einstein G, Fitzpatrick D (1991) Distribution and morphology of area 17 neurons that project to the cat's extrastriate cortex. J Comp Neurol 303:132-149.

Fuchs AF, Robinson DA (1966) A method for measuring horizontal and vertical eye movements of the trained cat. J Appl Physiol 21: 1068-1070.

Galli L, Chalupa L, Maffei L, Bisti S (1988) The organization of receptive fields in area 18 neurones of the cat varies with the spatiotemporal characteristics of the visual stimulus. Exp Brain Res 71:1-7.

Gizzi MS, Katz E, Movshon JA (1990) Spatial and temporal analysis by neurons in the representation of the central visual field in the cat's lateral suprasylvian visual cortex. Vis Neurosci 5:463-468.

Guido W, Spear PD, Tong L (1990) Functional compensation in the lateral suprasylvian visual area following bilateral visual cortex damage in kittens. Exp Brain Res 83:219-224.

Kimura M, Shiida T, Tanaka K, Toyama K (1980) Three classes of area 19 cortical cells of the cat classified by their neuronal connectivity and photic responsiveness. Vision Res 20:60-77.

Lehmkuhle S, Kratz K, Sherman SM (1982) Spatial and temporal sensitivity of normal and amblyopic cats. J Neurophysiol 48:372387.

Levinson E, Sekuler $\mathbf{R}$ (1975) The independence of channels in human vision selective for direction of movement. J Physiol (Lond) 250: 347-366.

Meyer G, Albus K (1981) Spiny stellates as cells of origin of association fibres from area 17 to area 18 in the cat's neocortex. Brain Res 210: 335-341.

Mignard M, Malpeli JG (1991) Paths of information flow through visual cortex. Science 251:1249-1251.

Morrone MC, DiStefano M, Burr D (1986) Spatial and temporal properties of neurons of the lateral suprasylvian cortex of the cat. J Neurophysiol 56:969-986.

Movshon J $\Lambda$, Thompson ID, Tolhurst DJ (1978) Spatial and temporal contrast sensitivity of neurones in areas 17 and 18 of the cat's visual cortex. J Physiol (Lond) 283:101-120.

Nault B, Michaud Y, Morin C, Casanova C, Molotchnikoff S (1990) Responsiveness of cells in area 17 after local interception of the descending path from area 18. Soc Neurosci Abstr 16:1219.

Nealey TA, Maunsell JHR, Merigan WH (1992) Visual function after a lesion of cortical area V2 in the macaque (abstract). Invest Ophthalmol Vis Sci 33:1130.

Newsome WT, Pare EB (1988) A selective impairment of motion perception following lesion of the middle temporal visual area (MT). J Neurosci 8:2201-2211.

Orban GA, Kennedy H, Maes H (1981a) Response of movement of neurons in areas 17 and 18 of the cat: velocity sensitivity. J Neurophysiol 45:1043-1058.

Orban GA, Kennedy H, Maes H (1981b) Response of movement of neurons in areas 17 and 18 of the cat: direction selectivity. J Neurophysiol 45:1059-1073.

Pasternak T (1986) The role of cortical directional selectivity in detection of motion and flicker. Vision Res 26:1187-1194.

Pasternak T (1987) Discrimination of differences in speed and flicker rate depends on directionally selective mechanisms. Vision Res 27 : $1881-1890$.

Pasternak T (1990) Vision following loss of cortical directional selectivity. In: Comparative perception, basic mechanisms, Vol 1 (Berkley M, Stebbins W, eds), pp 407-428. New York: Wiley.

Pasternak T, Horn K (1991) Spatial vision of the cat: variation with eccentricity. Vis Neurosci 6:151-158.

Pasternak T, Leinen LJ (1986) Pattern and motion vision in cats with selective loss of cortical directional selectivity. J Neurosci 6:938-945. 
Pasternak T Schumer RA Gizzi MS Movshon JA (1985) Abolition of cortical directional selectivity affects visual behavior in cats. Exp Brain Res 61:214-217.

Pasternak T, Maunsell JHR, Polashenski W, Merigan WH (1991) Deficits in global motion perception after MT/MST lesions in a macaque. Invest Ophthalmol Vis Sci [Suppl] 32:824.

Price DJ (1985) Patterns of cytochrome oxidase activity in areas 17 , 18 and 19 of the visual cortex of cats and kittens. Exp Brain Res 58: 125-133.

Remmel RS (1984) An inexpensive eye movement monitor using the scieral search coil technique. IEEE Trans Biomed Eng 31:388-390.

Sandell JH, Schiller PH (1982) Effect of cooling area 18 on striate cortex cells in the squirrel monkey. $J$ Neurophysiol 48:38-48.

Sparks DL, Sides JP (1974) Brain stem unit activity related to horizontal eye movements occurring during visual tracking. Brain Res $77: 320-325$.

Spear PD, Baumann TP (1975) Receptive-field characteristics of single ncurons in lateral suprasylvian visual area of the cat. J Neurophysiol 38:1403-1420.
Spear PD, Baumann TP (1979) Effects of visual cortex removal on receptive-field properties of neurons in lateral suprasylvian visual area of the cat. J Neurophysiol 42:31-56.

Symonds LL, Rosenquist AC (1984) Laminar origins of visual corticortical connections in the cat. J Comp Neurol 229:39-47.

Tanaka K, Ohzawa I, Ramoa AS, Freeman RD (1987) Receptive field properties of cells in area 19 of the cat. Exp Brain Res 65:549-558.

Tusa RJ, Rosenquist AC, Palmer LA (1979) Retinotopic organization of areas 18 and 19 in the cat. J Comp Neurol 185:657-678.

Watson AB, Thompson PG, Murphy BJ, Nachmias J (1980) Summation and discrimination of gratings moving in opposite directions. Vision Res 20:341-348.

Wong-Riley M (1979) Changes in the visual system of monocularly sutured or enucleated cats demonstrable with cytochrome oxidase histochemistry. Brain Res 171:11-28.

Yamasaki DS, Wurtz RH (1991) Recovery of function after lesions in the superior temporal sulcus in the monkey. $\mathrm{J}$ Neurophysiol 66: $651-673$. 